\title{
Fracture Toughness for Brittle Fracture of Elastic and Plastic Materials
}

\author{
Yoshikazu Tanabe \\ National Museum of Nature and Science, Tsukuba 305-0005, Japan
}

\begin{abstract}
The crack energy density causes a new expression of fracture toughness for brittle fracture, together with the concept of Barenblatt's characteristic distance $d_{\mathrm{c}}$ of cohesive zone at the edge of the crack. The Griffith's formula for fracture toughness is modified by the ratio of $\sigma_{\mathrm{T}} / \Sigma_{\mathrm{T}}$, which $\sigma_{\mathrm{T}}$ is the true fracture stress and $\Sigma_{\mathrm{T}}$ the maximum strength of the material obtainable by ordinary processing. The fracture toughness of elastic-plastic material, $K_{\mathrm{Ic}}^{*}$ for brittle fracture is described by the product of $\sigma_{\mathrm{T}}$ and the root of the observed absorption energy by Charpy impact test corrected for instrumental effect, $\sqrt{I_{\mathrm{cp}}^{\text {corrected }}}$. The characteristic distance $d_{\mathrm{c}}$ depends linearly on the released elastic energy, which is the physical basis of above-mentioned estimation formula. [doi:10.2320/matertrans.M2012348]
\end{abstract}

(Received October 18, 2012; Accepted December 10, 2012; Published January 25, 2013)

Keywords: fracture toughness, crack energy density, true fracture stress, corrected Charpy impact value, Barenblatt's characteristic distance

\section{Introduction}

For steels showing the unstable fracture (brittle fracture), Oda, Nakamura and Kawakami have reported before ca. 40 years $^{1,2)}$ that the fracture toughness $K_{\mathrm{Ic}}^{*}$ is related to the two observed values, the true fracture stress $\sigma_{\mathrm{T}}$ and the Charpy impact value $I_{\mathrm{cp}}^{\mathrm{obs}}$ as

$$
K_{\mathrm{Ic}}^{*}=k \sigma_{\mathrm{T}} \sqrt{\frac{I_{\mathrm{cp}}^{\mathrm{obs}}}{E}}
$$

where $E$ is the Young's modulus and $k$ a nondimensional constant. $^{1-3)}$ Equation (1) is important at two points, 1) that the fracture toughness $K_{\mathrm{Ic}}^{*}$ is described by the true fracture stress $\sigma_{\mathrm{T}}$ and Charpy impact value $I_{\mathrm{cp}}^{\mathrm{obs}}$ which are easily measured without any restriction of specimen size, and 2) that this equation can be used for the brittle fracture of materials with wide range of plastic deformation such as perfectly elastic, small scale yielding, large scale yielding and general yielding. T. Oda et al. have explained eq. (1) based upon the dimensional analysis. Trying to make clear the physical meaning of eq. (1) needs careful consideration because the well-known fracture mechanics is essentially based on elasticity not only in linear fracture mechanics but also in J-integral for larger scale yielding. In J-integral, the outside of integral contour must be elastic state. J-integral can be used in perfectly elastic state, small scale yielding and large scale yielding states but not in general yielding state. The method of analysis including general yielding is not known. This paper starts from the concept of crack energy density (CED) proposed by K. Watanabe. ${ }^{4-7)}$ The CED can be defined in any state of plastic deformation including general yielding.

The crack energy density is defined to be the area density of (strain) energy representing the work (per unit area) given at the point of crack edge from the outside of the crack in the plane including its point of the crack edge. The CED can be experimentally evaluated from the load-displacement curves as $\varepsilon=-(\partial U / \partial a)$, where total strain energy is $U(a, u)=\int P d u$, the load $P$, the displacement $u$ and the crack length $2 a$.

In elastic-plastic materials, CED is denoted by $\varepsilon=\varepsilon^{\mathrm{e}}+\varepsilon^{\mathrm{p}}$ where $\varepsilon^{\mathrm{e}}$ and $\varepsilon^{\mathrm{p}}$ are the parts of CED preserved elastically and plastically, respectively, during plastic deformation. ${ }^{6}$ The elastic CED, $\varepsilon^{\mathrm{e}}$ is released after unloading of the external force $P$. In the case of linear elastic materials, $\varepsilon=\varepsilon^{\mathrm{e}}=$ $\left(\left(1-v^{2}\right) / E\right) K_{\mathrm{I}}^{2}=G$ where $v$ is the Poisson's ratio, $K_{\mathrm{I}}$ the stress intensity factor, $G$ the energy release rate. Only the mode I (opening mode) of crack surface displacement is considered and discussion is made based on the twodimensional elasticity and the condition of plane strain, hereafter.

\section{Brittle Fracture of Perfectly Elastic Materials}

The problem of singularity at each crack tip in stress field is overcome by introducing the cohesive forces (or attraction) acting in small edge regions of crack, which is previously discussed by Barenblatt ${ }^{8)}$ and Goodier ${ }^{9)}$ in detail.

Tensile stress $\sigma_{\mathrm{y}}$ normal to the surface of a crack and the associated vertical displacement $v$ of the crack surface (y direction, see Fig. 1), are given by

$$
\begin{aligned}
\sigma_{\mathrm{y}} & =\frac{K_{\mathrm{I}}+K_{0}}{\sqrt{2 \pi s_{1}}}+g(0)+O\left(s_{1}^{\frac{1}{2}}\right) \\
v & = \pm 4 \frac{1-v^{2}}{E}\left(K_{\mathrm{I}}+K_{0}\right) \sqrt{\frac{s_{2}}{2 \pi}}+O\left(s_{2}^{\frac{3}{2}}\right)
\end{aligned}
$$

where \pm refers to upper and lower faces of displacement. The stress intensity factors $K_{\mathrm{I}}$ and $K_{0}$ are

$$
\begin{aligned}
K_{\mathrm{I}} & =2 \sqrt{\frac{a}{\pi}} \int_{0}^{a} \frac{T_{0}(t)}{\sqrt{a^{2}-t^{2}}} d t \\
K_{0} & =-\sqrt{\frac{2}{\pi}} \int_{0}^{d} \frac{g(\xi)}{\sqrt{\xi}} d \xi
\end{aligned}
$$

and $T_{0}(t)$ is the distribution of applied loads, $g(\xi)$ the distribution of cohesive forces near the crack tip, $2 a$ the length of crack, $x=a-t$ where $t$ is a running coordinate on the $x$ axis, $s_{1}=x-a, s_{2}=a-x$ and $d$ is the distance for acting cohesive forces (see Fig. 1). The stress singularity occurs from the term $s_{1}^{-\frac{1}{2}}$ in eq. (2), and the postulate $K_{\mathrm{I}}+K_{0}=0$ by Barenblatt $^{8)}$ and Goodier ${ }^{9)}$ removes its singularity. The remaining main terms in eq. (3) are of order $s_{2}^{\frac{3}{2}}$, which is demonstrated in Fig. 1 .

When the cohesive force distribution exists near the crack edge (tip), the crack energy density $\varepsilon$ at one end of crack is the work done near the crack edge $(W)$ during an infinitesimal extension of the crack. 


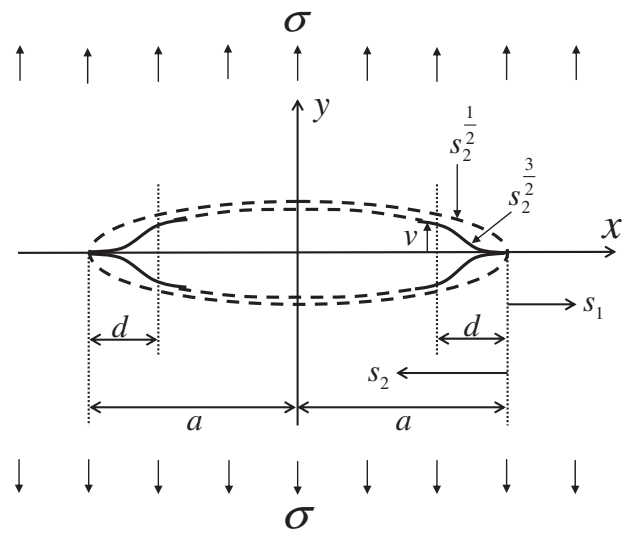

Fig. 1 Vertical displacement of the crack faces under the condition of finite stress at the edge of crack (crack tip).

$$
\varepsilon=\frac{\mathrm{d} W}{\mathrm{~d} a}=\frac{2}{\pi}\left(\frac{1-v^{2}}{E}\right)\left[\int_{0}^{d} \frac{g(\xi)}{\sqrt{\xi}} \mathrm{d} \xi\right]^{2}
$$

which can be derived from Goodier's result by exchanging $g_{\mathrm{m}}(\xi)$ for $g(\xi)$, where $g_{\mathrm{m}}(\xi)$ is the limiting cohesive force distribution. (Goodier' ${ }^{9}$ used $G$ for $g$ ) The distance $d$ is the size of the cohesive zone. Using eq. (5),

$$
\varepsilon=\frac{1-v^{2}}{E} K_{0}^{2}
$$

Barenblatt suggests that cracks in perfectly elastic materials extend, when all the work of the cohesive forces is equal to new surface energy $2 \gamma$. Here the critical value of energy release rate $G_{\mathrm{c}}$ is used instead of $2 \gamma$.

$$
\varepsilon_{\mathrm{c}}=\frac{1-v^{2}}{E} K_{0 \mathrm{c}}^{2}=G_{\mathrm{c}}
$$

where $\varepsilon_{\mathrm{c}}$ and $K_{0 \mathrm{c}}$ is the critical value of $\varepsilon$ and $K_{0}$, respectively.

When the distribution of external force (applied load) $T_{0}(x)$ exists without the cohesive force distribution $g(\xi)$, the crack energy density $\varepsilon$ is calculated as the apparent work done on the body. The crack $2 a$ is assumed to extend as $\delta a$ infinitesimally,

$$
\varepsilon=\frac{1}{\delta a} 2 \int_{0}^{\delta a} \frac{1}{2} \sigma_{\mathrm{y}} v d x=\frac{1-v^{2}}{E} K_{\mathrm{I}}^{2}
$$

which is the well-known result with Griffith ${ }^{10)}$ and many other authors. ${ }^{11-13)}$ In Griffith's theory, the critical energy $\varepsilon_{\mathrm{c}}$ for perfectly elastic materials is assigned to the surface energy $2 \gamma_{\mathrm{s}}$.

$$
\varepsilon_{\mathrm{c}}=\frac{1-v^{2}}{E} K_{\mathrm{Ic}}^{2}=2 \gamma_{\mathrm{s}}
$$

where $K_{\mathrm{Ic}}$ is usually called fracture toughness.

Barenblatt and Goodier has made the postulate $K_{\mathrm{I}}+$ $K_{0}=0$ in order to remove the stress singularity at the crack edges. ${ }^{8,9)}$ This also shows eqs. (7) and (9) give the same $\varepsilon$, and it is natural that $\varepsilon_{\mathrm{c}}$ in eqs. (8) and (10) gives the same surface energy $2 \gamma_{\mathrm{s}}$ or critical energy release rate $G_{\mathrm{c}}$. These relations including the postulate $K_{\mathrm{I}}+K_{0}=0$ are selfconsistent in the framework of linear elasticity for the perfectly elastic materials. And the following relation is noted using $G_{\mathrm{c}}$ instead of $2 \gamma_{\mathrm{s}}$,

$$
K_{\mathrm{Ic}}=\left|K_{0 \mathrm{c}}\right|=\sqrt{\frac{E G_{\mathrm{c}}}{1-v^{2}}}
$$

The critical energy release rate or the surface energy depends on the mechanisms of breaking(rupture) and reflects the number of effective bonding which depends on very small voids, cracks, defects, impurities, dislocations and so on.

To compare with experimental results, the information of distribution of $T_{0}(t)$ and $g(\xi)$ is necessary. The simplest case is the uniform distribution of external force (applied load) and $T_{0}(t)=\sigma(\sigma$ : external tensile stress). The distribution of $g(\xi)$ is not yet known, and the limiting cohesive force distribution $g_{\mathrm{c}}(\xi)$ which is given by $K_{0 \mathrm{c}}=-\sqrt{2 / \pi} \int_{0}^{d} g_{\mathrm{c}}(\xi) / \sqrt{\xi} \mathrm{d} \xi$, is assumed as $g_{\mathrm{c}}(\xi)=\sigma_{\mathrm{T}}$ where $\sigma_{\mathrm{T}}$ is the tensile strength of the perfectly elastic materials. This assumption seems to be reasonable as the zeroth approximation of $g_{c}(\xi)$ because the crack extends if atomic or molecular attraction cannot hold bonding. It means cohesive forces cannot become larger than $\sigma_{\mathrm{T}}$ and the critical value of cohesive forces is approximated by $\sigma_{\mathrm{T}}$. Of course, this is very rough estimation and only the starting point of discussion of $g(\xi)$.

From $T_{0}(t)=\sigma$,

$$
K_{\mathrm{I}}=\sigma \sqrt{\pi a}
$$

which is the well-known expression of stress intensity factor. ${ }^{11-13)}$ From $g_{\mathrm{c}}(\xi)=\sigma_{\mathrm{T}}$

$$
\left|K_{0}\right|=\sigma_{\mathrm{T}} \sqrt{\pi d_{\mathrm{c}}}
$$

and

$$
d_{\mathrm{c}}=\frac{8}{\pi^{2}} d
$$

where $d_{\mathrm{c}}$ is the characteristic distance of cohesive zone. Equation (13) is the same form as eq. (12) by using $d_{\mathrm{c}}$.

For a kind of perfectly elastic material with the largest strength after various plastic processing (a kind of extreme state),

$$
\left|K_{0 \mathrm{c}}^{\mathrm{high}}\right|=\Sigma_{\mathrm{T}} \sqrt{\pi d_{\mathrm{c}}}=\sqrt{\frac{E G_{\mathrm{c}}^{\mathrm{high}}}{1-v^{2}}}
$$

where $\Sigma_{\mathrm{T}}$ is the maximum strength of the material. As Griffith has previously shown, ${ }^{10)}$ glass has the extreme state and $G_{\mathrm{c}}^{\text {high }}$ in eq. (15) is equal to the surface energy $2 \gamma_{\mathrm{s}}$. For steels, the realization of extreme state is very difficult by the ordinary processing method such as forging and pressing.

For the perfectly elastic material after ordinary processing (not extreme but ordinary state),

$$
\left|K_{0 \mathrm{c}}^{\text {low }}\right|=\sigma_{\mathrm{T}} \sqrt{\pi d_{\mathrm{c}}}=\sqrt{\frac{E G_{\mathrm{c}}^{\text {low }}}{1-v^{2}}}
$$

where the critical energy release rate $G_{\mathrm{c}}^{\text {low }}$ is smaller than $G_{\mathrm{c}}^{\text {high }}$. For example, if very small cracks exist within the distance $d_{c}$, this sample is perfectly elastic and has lower tensile strength $\sigma_{\mathrm{T}}$ than $\Sigma_{\mathrm{T}}$.

If eq. (15) is assumed to be the reference state of the material with the same geometry (same $d_{\mathrm{c}}$ ), eqs. (15) and (16) derive

$$
K_{\text {Ic }}^{\text {low }}=\left|K_{0 \mathrm{c}}^{\text {low }}\right|=\frac{\sigma_{\mathrm{T}}}{\Sigma_{\mathrm{T}}} \sqrt{\frac{E G_{\mathrm{c}}^{\text {high }}}{1-v^{2}}}
$$


where $\sigma_{\mathrm{T}} / \Sigma_{\mathrm{T}}=\sqrt{G_{\mathrm{c}}^{\text {low }} / G_{\mathrm{c}}^{\text {high }}}$. Equations (12) and (17) give

$$
\sigma=\frac{\sigma_{\mathrm{T}}}{\Sigma_{\mathrm{T}}} \sqrt{\frac{E G_{\mathrm{c}}^{\mathrm{high}}}{\pi\left(1-v^{2}\right) a}}
$$

which is the breaking stress with a crack of $2 a$. This equation is different in the term of $\sigma_{\mathrm{T}} / \Sigma_{\mathrm{T}}$ from the usual Griffith's formula. ${ }^{10-13)}$ The factor $\sigma_{\mathrm{T}} / \Sigma_{\mathrm{T}}=\sqrt{G_{\mathrm{c}}^{\text {low }} / G_{\mathrm{c}}^{\text {high }}}$ causes the difference of fracture toughness between well-processed extreme state and less processed ordinary one. The factor $\sigma_{\mathrm{T}} / \Sigma_{\mathrm{T}}$ describes the difference of the stored elastic energy at the break in perfectly elastic materials.

\section{Fracture Toughness $K_{\text {Ic }}^{*}$ of Elastic-Plastic Solids}

The CED of elastic-plastic materials for the mode I of crack surface displacement is

$$
\varepsilon=\varepsilon^{\mathrm{e}}+\varepsilon^{\mathrm{p}}=\frac{1-v^{2}}{E} K_{\mathrm{I}}^{2}+\varepsilon^{\mathrm{p}}
$$

where $\varepsilon^{\mathrm{e}}$ and $\varepsilon^{\mathrm{p}}$ are the elastic and plastic parts of CED, respectively, and $\varepsilon^{\mathrm{e}}=\left(\left(1-v^{2}\right) / E\right) K_{\mathrm{I}}^{2}=G$ where $G$ is the energy release rate. The nonlinear part of $\varepsilon^{\mathrm{e}}$ is neglected. If the critical value of $\varepsilon$ in the right-hand side of eq. (19) is denoted by $\varepsilon_{\mathrm{c}}=\left(\left(1-v^{2}\right) / E\right) K_{\mathrm{Ic}}^{* 2}, \quad K_{\mathrm{Ic}}^{*}$ is a kind of extended fracture toughness since $\varepsilon_{\mathrm{c}}$ is the energy per unit area for breaking(rupture) of the crack edge. The parameter $K_{\text {Ic }}^{*}$ is the redefined fracture toughness containing the effect of plastic deformation. In perfectly elastic materials, $K_{\mathrm{Ic}}^{*}$ is the same as the fracture toughness $K_{\text {Ic }}$ and related to the energy release rate $G$. The redefined fracture toughness $K_{\mathrm{Ic}}^{*}$ is given as

$$
K_{\mathrm{Ic}}^{* 2}=K_{\mathrm{Ic}}^{2}+\left(\frac{E}{1-v^{2}}\right) 2 \gamma_{\mathrm{p}}
$$

where $\varepsilon_{\mathrm{c}}^{\mathrm{p}}=2 \gamma_{\mathrm{p}}$ as described in various publications. ${ }^{11-13)}$ Using eq. (17),

$$
K_{\mathrm{Ic}}^{*}=\frac{\sigma_{\mathrm{T}}}{\Sigma_{\mathrm{T}}} \sqrt{\left(\frac{E}{1-v^{2}}\right)\left[G_{\mathrm{c}}^{\mathrm{high}}+\left(\frac{\Sigma_{\mathrm{T}}}{\sigma_{\mathrm{T}}}\right)^{2} 2 \gamma_{\mathrm{p}}\right]}
$$

where $G_{\mathrm{c}}^{\text {high }}$ is the stored elastic energy in the material after plastic deformation, and is also the released energy. In eq. $(21), K_{\mathrm{Ic}}=\left(\sigma_{\mathrm{T}} / \Sigma_{\mathrm{T}}\right) \sqrt{\left(E G_{\mathrm{c}}^{\text {high }} /\left(1-v^{2}\right)\right)}$ is related to the state of materials just before breaking(rupture) after plastic deformation finishes (see eq. (17)). Equation (21) is applicable for all elastic-plastic materials independent of degree of plasticity.

When $G_{\mathrm{c}}^{\text {low }} \gg 2 \gamma_{\mathrm{p}}\left(\right.$ or $\left.G_{\mathrm{c}}^{\text {high }} \gg\left(\frac{\Sigma_{\mathrm{T}}}{\sigma_{\mathrm{T}}}\right)^{2} 2 \gamma_{\mathrm{p}}\right)$, in which brittle fracture occurs,

$$
K_{\mathrm{Ic}}^{*} \cong \frac{\sigma_{\mathrm{T}}}{\Sigma_{\mathrm{T}}} \sqrt{\left(\frac{E}{1-v^{2}}\right) G_{\mathrm{c}}^{\text {high }}}
$$

and when $G_{\mathrm{c}}^{\text {low }} \ll 2 \gamma_{\mathrm{p}}$ (or $\left.G_{\mathrm{c}}^{\text {high }} \ll\left(\frac{\Sigma_{\mathrm{T}}}{\sigma_{\mathrm{T}}}\right)^{2} 2 \gamma_{\mathrm{p}}\right)$, in which ductile fracture occurs,

$$
K_{\mathrm{Ic}}^{*}=\sqrt{\left(\frac{E}{1-v^{2}}\right)\left(G_{\mathrm{c}}^{\text {low }}+2 \gamma_{\mathrm{p}}\right)} \cong \sqrt{\left(\frac{E}{1-v^{2}}\right) 2 \gamma_{\mathrm{p}}}
$$

Equation (22) shows that the structure of the material changes through plastic deformation although the plastic energy is very small, and the elastic energy is stored as $G_{\mathrm{c}}^{\text {high }}$ which is larger than $G_{\mathrm{c}}^{\text {low }}$. This is experimentally verified by the result that the larger elastic energy state appears by unloading just before the break(rupture).

The fracture toughness $K_{\text {Ic }}$ appearing in eq. (20), is related to the linear part of preserved elastic CED just before breaking(rupture). Here, it is implicitly assumed that the breaking of materials such as metals occurs only at the condition of perfectly elastic state. In other words, metals undertake the plastic deformation until the perfectly elastic state appears. The appearance of perfectly elastic state after the plastic deformation, causes the Griffith's breaking. The reason why the perfectly elastic state appears after plastic deformation, is that the plastic deformation continues until the moving units in materials vanish. The state that the moving units vanish, is the perfectly elastic one. Breaking occurs only in the perfectly elastic state.

Equation (23) is directly derived from eq. (19). This is well-known result as Griffith ${ }^{10)}$-Irwin ${ }^{14)}$-Orowan ${ }^{15)}$ formula. Compared with eq. (22), eq. (23) has no effect of the factor $\sigma_{\mathrm{T}} / \Sigma_{\mathrm{T}}$. The difference of stored elastic energy causes the factor $\sigma_{\mathrm{T}} / \Sigma_{\mathrm{T}}$ and does not affect the plastic part of $2 \gamma_{\mathrm{p}}$.

\section{Estimation of Absorbed Energy by Experimentally Observable Parameter}

The exact method to observe the absorbed energy relating to the static fracture toughness is not yet known. The surface energy $2 \gamma_{\mathrm{s}}$ discussed by Griffith and many authors is effective concept only in single crystals and amorphous materials. The absorbed energy can be approximately observed by impact tests under some conditions. The observed Charpy impact value $I_{\mathrm{cp}}^{\text {obs }}$ contains $G_{\mathrm{c}}^{\text {high }}+2 \gamma_{\mathrm{p}}$ together with the stored energy in the instrument and the toss energy of broken specimen. The absorption energy is related to Charpy impact value as $G_{\mathrm{c}}^{\text {high }}+2 \gamma_{\mathrm{p}}=\alpha I_{\mathrm{cp}}^{\text {obs }}=I_{\mathrm{cp}}^{\text {corrected }}$ where the correction parameter $\alpha$ is $0<\alpha<1$ and $\alpha$ must be determined from experimental system. If the observed impact value $I_{\mathrm{cp}}^{\mathrm{obs}}$ contains only the absorption energy by deformation and fracture, $\alpha=1$. Usually, $\alpha$ includes other effects such as elastic deformation of testing machine and toss energy of broken specimen. Kobayashi et al. ${ }^{16,17)}$ have shown the method to measure the correction parameter $\alpha$ by the instrumented impact testing method, and report that $\alpha$ is about 0.8 for steel and 0.5 for cast iron. ${ }^{16)}$ Brittle materials like ceramics reveal rather lower values of $\alpha$ as $0.4-0.6{ }^{17)}$

It must be noted that the observed Charpy impact value generally reflects the mechanism of dynamic fracture toughness and is not always related to the mechanism of static fracture toughness discussed in the above-mentioned sections. This means that the absorbed energy for static fracture toughness would be different from the dynamic case. In the low-velocity (low blow) impact tests in the instrumented Charpy impact testing system, the dynamic fracture toughness is nearly equal to the static one for steel within the error of measurement, which suggests $\alpha$ is correctly estimated. ${ }^{18)}$ In the high-velocity impact test, other discussion must be needed including the effect of loss of contact and is beyond the reach of this paper.

In eq. (21), $G_{\mathrm{c}}^{\text {high }}$ appears. Even small plastic deformation causes the change of structure of the material and the elastic energy $G_{\mathrm{c}}^{\text {low }}$ increases to $G_{\mathrm{c}}^{\text {high }}$. This $G_{\mathrm{c}}^{\text {high }}$ is observed by 
low-velocity impact tests in the case of brittle fracture. Impact tests include all energy as elastic and inelastic energies until the break occurs, and also reflect the increase of elastic energy through structural change caused by plastic deformation. If inelastic energy $2 \gamma_{\mathrm{p}}$ is very small, $G_{\mathrm{c}}^{\text {high }}$ is approximated by $I_{\mathrm{cp}}^{\text {corrected }}$.

When $G_{\mathrm{c}}^{\text {low }} \gg 2 \gamma_{\mathrm{p}}$, in which brittle fracture occurs,

$$
K_{\mathrm{Ic}}^{*} \cong \frac{\sigma_{\mathrm{T}}}{\Sigma_{\mathrm{T}}} \sqrt{\left(\frac{E}{1-v^{2}}\right) I_{\mathrm{cp}}^{\text {corrected }}}
$$

and when $G_{\mathrm{c}}^{\text {low }} \ll 2 \gamma_{\mathrm{p}}$, in which ductile fracture occurs,

$$
K_{\mathrm{Ic}}^{*} \cong \sqrt{\left(\frac{E}{1-v^{2}}\right) I_{\mathrm{cp}}^{\text {corrected }}}
$$

These equations (eqs. (24) and (25)) are the estimation formula of fracture toughness by using easily observable parameters $\sigma_{\mathrm{T}}$ and $I_{\mathrm{cp}}^{\text {corrected }}$. The parameter $\Sigma_{\mathrm{T}}$ is the maximum strength of materials achieved by processing plastically. It is well known that the ordinary deformation process leads a kind of upper limit of mechanical strength which is about ten $\%$ of ideal mechanical strength. This upper limit is $\Sigma_{\mathrm{T}}$ and steel shows $\Sigma_{\mathrm{T}}$ as ca. $2 \mathrm{GPa}$ (for example, piano wire). Ordinary processing techniques such as forging, rolling, pressing and heat treatment cannot reveal the ideal mechanical strength (ideal cleavage strength) because those techniques cannot control the fine structure of materials below the size of a few hundreds of nanometer. In other words, $\Sigma_{\mathrm{T}}$ is the maximum mechanical strength of the specimen in case that its fine structure above a few hundreds of nanometer is precisely controlled. ${ }^{11,12,16)}$

The fracture toughness $K_{\mathrm{Ic}}^{*}$ in eq. (24) includes the two effects, one is the effect of absorption energy $I_{\mathrm{cp}}^{\text {corrected }}$ through $\sqrt{\left(E I_{\mathrm{cp}}^{\text {corrected }} /\left(1-v^{2}\right)\right)}$ with Young's modulus $E$ and the other is the effect of the true fracture stress $\sigma_{\mathrm{T}}$. If $\sigma_{\mathrm{T}}=\Sigma_{\mathrm{T}}$ (in the case of perfectly elastic and extremely well-processed materials), eq. (24) is equal to the Griffith's formula (eq. (11)) because of $I_{\mathrm{cp}}^{\text {corrected }}=G_{\mathrm{c}}=2 \gamma_{\mathrm{s}}$.

Equation (24) is derived based on the CED consideration and the assumption that the break(rupture) of materials occurs at the perfectly elastic state even after the plastic deformations. Equation (24) can be used not only in perfectly elastic materials but in general yielding because of no restriction of degree of plasticity, although the energy condition $G_{\mathrm{c}}^{\text {low }} \gg 2 \gamma_{\mathrm{p}}$ must be maintained. The estimation formula of fracture toughness, eq. (24) has physical base and can be used to estimate the fracture toughness from easily measurable parameters, absorption energy $I_{\mathrm{cp}}^{\text {corrected }}$ and true tensile strength $\sigma_{\mathrm{T}}$.

\section{Discussion}

From eqs. (15) and (16),

$$
d_{\mathrm{c}}=\frac{1}{\pi}\left(\frac{K_{\mathrm{Ic}}^{\text {low }}}{\sigma_{\mathrm{T}}}\right)^{2}=\frac{E}{\pi\left(1-v^{2}\right)} \frac{1}{\Sigma_{\mathrm{T}}^{2}} G_{\mathrm{c}}^{\mathrm{high}}
$$

This equation means that the characteristic distance $d_{\mathrm{c}}$ is proportional to $G_{\mathrm{c}}^{\text {high }}$ for brittle materials. The eq. (26) is the same as eq. (22). As described before, $G_{\mathrm{c}}^{\text {high }}$ is measured by $I_{\mathrm{cp}}^{\text {corrected }}$, and $d_{\mathrm{c}}$ is calculated from $K_{\mathrm{Ic}}^{\text {low }}$ and $\sigma_{\mathrm{T}}$. Figure 2 is the replotting of data by Oda et al., ${ }^{1,2)}$ although they have not

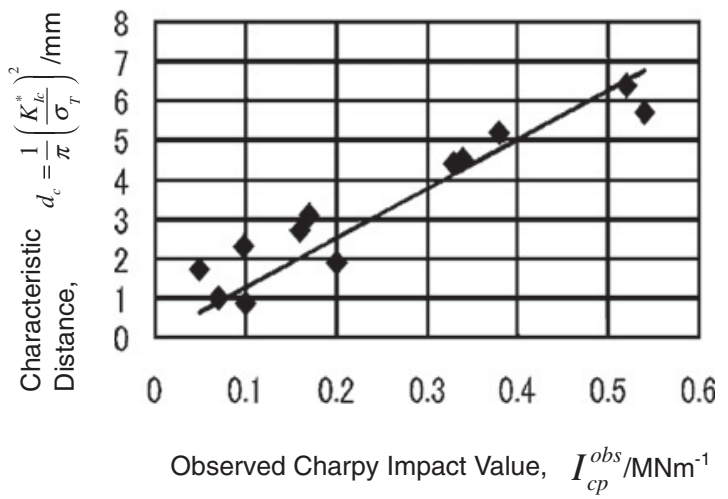

Fig. 2 The relationship between the Barenblatt's characteristic distance $d_{\mathrm{c}}$ and observed Charpy impact value $I_{\mathrm{cp}}^{\mathrm{obs}}$, in which data used are by Oda et al. ${ }^{1,2)}$

corrected the instrumental effect of impact test but its effect $\sqrt{\alpha}$ is rather small in steels. It is clear that $d_{\mathrm{c}}$ depends on $I_{\mathrm{cp}}^{\mathrm{obs}}$ linearly. And the obtained slope $12.5(\mathrm{GPa})^{-1}$ gives ca. $2 \mathrm{GPa}$ for $\Sigma_{\mathrm{T}}$, which is reasonable one. This result denotes that $G_{\mathrm{c}}^{\text {high }}$ is described as $G_{\mathrm{c}}^{\text {high }} \approx\left(\Sigma_{\mathrm{T}}^{2} / 2 E\right) d_{\mathrm{c}}$. The strain energy $\Sigma_{\mathrm{T}}^{2} / 2 E$ per unit volume gathered in the range of $d_{\mathrm{c}}$, gives $G_{\mathrm{c}}^{\text {high }}$, which is the elastic energy released by breaking. It is not clear whether the value of $G_{\mathrm{c}}^{\text {high }}$ would coincide with the surface energy or not. In addition, the result that $\Sigma_{\mathrm{T}}$ is obtained from the slope in Fig. 2, suggests that the elastic state just before breaking is the one with the tensile strength $\Sigma_{\mathrm{T}}$ at least in the range of $d_{\mathrm{c}}$. Moreover, the larger $d_{\mathrm{c}}$, the larger the fracture toughness. The eq. (24) is based on that $d_{\mathrm{c}}$ depends linearly on $G_{\mathrm{c}}^{\text {high }}$.

Equation (24) is important because the fracture toughness $K_{\text {Ic }}^{*}$ can be estimated from the observed physical values as true fracture stress $\sigma_{\mathrm{T}}$, Young's modulus $E$ and the absorption energy $I_{\mathrm{cp}}^{\text {corrected }}$ which can be measured easily and without using excessively large specimen.

The apparent maximum strength of specimen, $\Sigma_{\mathrm{T}}$ seems to be a kind of physical parameters because bulk materials such as metals and ceramics can be controlled only over the region of several hundreds of nanometer by ordinary processing methods. ${ }^{19)}$ The value of $\Sigma_{\mathrm{T}}$ can be observed on the bulk materials with precisely controlled fine structure over the range of a few hundreds of nanometer.

The previously proposed estimation formula, eq. (1) has apparently a different form from eq. (24). Compared with eqs. (1) and (24), the nondimensional constant $k$ is given by

$$
k=\frac{E}{\sqrt{1-v^{2}}} \frac{\sqrt{\alpha}}{\Sigma_{\mathrm{T}}}
$$

The constant $k$ is represented by the ratio of Young's modulus $E$ and the maximum strength of materials $\Sigma_{\mathrm{T}}$ reachable by plastic processing. In the case of steel, $E$ is ca. $200 \mathrm{GPa}, v$ is ca. $0.3, \Sigma_{\mathrm{T}}$ ca. $2 \mathrm{GPa}$ and $\alpha$ ca. $0.8,{ }^{15)}$ and $k$ is ca. 94 which is a similar value as shown by Oda et al. ${ }^{1,2)}$ and Nakamura et $a l .{ }^{3)}$ It must be noted that Oda et al. have not corrected the observed Charpy impact values but the effect of $\sqrt{\alpha}$ is rather small. Equation (24) is better for use than eq. (1) because it has clear physical meaning. And eq. (24) is important in the brittle fracture and cannot be used in the case of ductile fracture, as seen from the difference between eqs. (24) and (25). 


\section{Conclusions}

The concept of crack energy density (CED) is the base of the discussion of fracture phenomena of elastic-plastic solids such as metallic materials. The fracture toughness is redefined as $\varepsilon_{\mathrm{c}}=\left(\left(1-v^{2}\right) / E\right) K_{\mathrm{Ic}}^{* 2}$, where $\varepsilon_{\mathrm{c}}$ is the critical value of CED and the undertaking energy per unit area at the crack edge to break.

In brittle fracture of perfectly elastic materials, modified Griffith's formula of fracture toughness (eq. (17)) and breaking stress with a crack $2 a$ (eq. (18)) is obtained by taking into account of the Barenblatt's characteristic distance $d_{\mathrm{c}}$ at the edge of crack (tip).

The general expression of fracture toughness of elasticplastic solids is given by eq. (21). Equation (21) is applicable to all materials independent of degree of plasticity because its derivation is based on the energy consideration without expecting detailed mechanisms of elastic and/or plastic deformations and fracture.

For the brittle fracture of elastic-plastic materials, eq. (22) is derived and this equation causes the new estimation formula (eq. (24)) which can be used for brittle fracture regardless of the deformation such as perfectly elastic, small scale yielding, large scale yielding and general yielding under the condition of larger elastic energy than plastic energy.

The points are that the break(rupture) of bulk materials occurs at the perfectly elastic state after the plastic deformation, which is described by Griffith's mechanism, and the elastic energy stored in the material after plastic deformation is different from the initial one because of structural change. This elastic energy $G_{\mathrm{c}}^{\text {high }}$ can be observed by the low-velocity impact test of instrumented Charpy impact test, although some correction is needed for instrumental contribution.

The new estimation formula of fracture toughness, eq. (24) is of practical use since it is possible to estimate with easily measurable parameters such as $I_{\mathrm{cp}}^{\text {corrected }}$ and $\sigma_{\mathrm{T}}$, without using the remarkably large size specimen.

\section{Acknowledgments}

Sincere thanks are due to Mr. Makoto Nakamura, (formerly) Mitsubishi Heavy Industries, Ltd. for the valuable discussions during the course of this research and also due to Mr. Seiji Fukuda, (formerly) Mitsubishi Heavy Industries, Ltd. for offering a lot of information.

\section{REFERENCES}

1) T. Oda, M. Nakamura and H. Kawakami: J. Jpn. Inst. Met. 39 (1975) 725-735 (in Japanese).

2) T. Oda, M. Nakamura and H. Kawakami: Mitsubishi Heavy Industries Technical Rev. 12 (1975) 100-107 (in Japanese)

3) M. Nakamura and Y. Tanabe: Bull. Natl. Mus. Nat. Sci., E 31 (2008) 15-24 (in Japanese).

4) K. Watanabe: Trans. Jpn. Soc. Mech. Eng. 51 (1985) 873-882 (in Japanese).

5) K. Watanabe: Trans. Jpn. Soc. Mech. Eng. 47 (1981) 406-415 (in Japanese).

6) K. Watanabe: Trans. Jpn. Soc. Mech. Eng. 48 (1982) 1226-1236 (in Japanese).

7) K. Watanabe: Prep. Jpn. Soc. Mech. Eng. No. 804-1 (1980) 91-94 (in Japanese).

8) G. I. Barenblatt: Adv. Appl. Mech. 7 (1962) 55-129.

9) J. N. Goodier: Fracture, An Advance Treatise, (ed. H. Liebowitz, Academic Press, 1968), vol. II (Mathematical Fundamentals), Chap. 1.

10) A. A. Griffith: Proc. 1st Intern. Congr. Appl. Mech., Delft (1924) pp. 55-63.

11) H. Okamura: Introduction to Linear Fracture Mechanics, (Baifukan Co., Ltd., 2006) (in Japanese).

12) M. Shiratori, T. Miyoshi and H. Matsushita: Computational Fracture Mechanics, (Jikkyo Shuppan Co., Ltd., 1999) (in Japanese).

13) T. L. Anderson: Fracture Mechanics-Fundamental and Applications-, (CRC Press, Taylor \& Francis, 2005).

14) G. Irwin: Fracturing of Metals, (American Society for Metals, 1948) pp. 147-166.

15) E. Orowan: Fatigue and Fracture of Metals, (ed. W. M. Murray, Wiley, 1950) pp. 139-167.

16) T. Kobayashi and I. Yamamoto: Bull. Jpn. Inst. Met. 32 (1993) 151159 (in Japanese).

17) T. Kobayashi, M. Niinomi, Y. Koide and K. Matsunuma: Trans. JIM 27 (1986) 775-783.

18) M. Asano, T. Kobayashi, M. Nawa and K. Niihara: J. Jpn. Inst. Met. 60 (1996) 1222-1228 (in Japanese).

19) N. Tsuji: Tetsu-to-Hagane 88 (2002) 359-369 (in Japanese). 